TITLE:

\title{
Multitarget super-resolution microscopy with high-density labeling by exchangeable probes.
}

\section{$\operatorname{AUTHOR}(\mathrm{S}):$}

Kiuchi, Tai; Higuchi, Makio; Takamura, Akihiro; Maruoka, Masahiro; Watanabe, Naoki

\section{CITATION:}

Kiuchi, Tai ...[et al]. Multitarget super-resolution microscopy with highdensity labeling by exchangeable probes.. Nature methods 2015, 12: 743-746

ISSUE DATE:

2015-07-06

URL:

http://hdl.handle.net/2433/198729

\section{RIGHT:}

(c) 2015 Nature America, Inc.; 許諾条件により本文ファイルは2016-0106に公開.; This is not the published version. Please cite only the published version.; この論文は出版社版でありません。引用の際には 出版社版をご確認ご利用ください。 


\section{Multitarget super-resolution microscopy with high density labeling by exchangeable probes}

Tai Kiuchi ${ }^{1}$, Makio Higuchi' ${ }^{1}$, Akihiro Takamura ${ }^{2}$, Masahiro Maruoka ${ }^{2,4}$ and Naoki Watanabe ${ }^{1,3}$

${ }^{1}$ Department of Pharmacology, Kyoto University Faculty of Medicine, Kyoto, Japan

${ }^{2}$ Laboratory of Single-Molecule Cell Biology, Tohoku University Graduate School of Life Sciences, Sendai, Japan

${ }^{3}$ Laboratory of Single-Molecule Cell Biology, Kyoto University Graduate School of Biostudies, Kyoto, Japan

${ }^{4}$ Present address: Division of Pathogenetic Signaling, Department of Biochemistry and Molecular Biology, Kobe University Graduate School of Medicine, Kobe, Japan

Correspondence should be addressed to T.K. (kiuchi.tai.6z@kyoto-u.ac.jp) or N.W. (watanabe.naoki.4v@kyoto-u.ac.jp) 
We have developed a multitarget super-resolution microscopy technique called image reconstruction by integrating exchangeable single-molecule localization (IRIS). IRIS uses protein fragment-based probes that directly associate with and dissociate from their targets over durations on the order of tens of milliseconds. By integrating single-molecule localization and sequential labeling, IRIS enables unprecedented labeling density along multiple cellular structures. IRIS can be used to discern the area-specific proximity between cytoskeletal components and focal adhesions within a single cell.

Super-resolution fluorescence microscopy approaches, including stimulated emission depletion microscopy, structured illumination microscopy, photoactivated localization microscopy (PALM) and stochastic optical reconstruction microscopy (STORM), have overcome the resolution limit of light microscopy $(\sim 200 \mathrm{~nm})^{1,2}$. At a resolution of $\sim 20$ $\mathrm{nm}$, however, the accuracy of target localization is also limited by the density of labels on the targets ${ }^{3,4}$. Theoretically, according to the Nyquist-Shannon sampling theorem ${ }^{3-5}$, a distribution feature smaller than twice the distance between two labeled targets cannot be discerned. The labeling density of a fluorescent protein and an antibody on their targets may be limited owing to the expression level and the antibody size $(>10 \mathrm{~nm})^{4,6}$. The labeling density is also important in multicolor super-resolution imaging. Recently, Exchange-PAINT (a derivative of the point accumulation for imaging in nanoscale topography (PAINT) method) was shown to allow super-resolved imaging of multiple targets by sequentially hybridizing short, fluorescently labeled DNA with its complementary DNA conjugated to antibodies ${ }^{7}$. However, the use of multiple antibodies for multiplexed imaging may lead to spatial interference between the antibodies in a resolved area, which could result in mislocalization of the targets in the reconstructed images. Therefore, high-density labeling of each target that is essential for super-resolution imaging is not readily achievable using available methods ${ }^{3,4,6}$.

Here we introduce a new high-density labeling technique, IRIS, for multitarget super-resolution microscopy (Fig. 1a). IRIS is related to PALM and STORM microscopy. In PALM and STORM, photoactivatable or photoswitchable fluorescent probes that are tightly bound to target structures are individually visualized by stochastic photoactivation or photoswitching, and the central position of each probe is determined with high accuracy by fitting its photon distribution with the point spread function ${ }^{8,9}$. A huge number of images are used to reconstruct high resolution images by integrating such position information. Instead of photoactivatable or photoswitchable probes, IRIS employs exchangeable probes that repeatedly associate with and dissociate 
from their endogenous targets. This exchangeable single-molecule labeling by IRIS probes enables high density labeling of the targets, which is required for high-fidelity target localization at a resolution of $\sim 20 \mathrm{~nm}$. Moreover, IRIS can be readily extended to multiplexed imaging because of the exchangeable nature of the probes.

We tested whether the IRIS method yielded super-resolution images using Lifeact, a widely used actin marker. Lifeact is a short peptide that stains actin filaments in live and fixed cells ${ }^{10}$. Lifeact exchanges within $0.4 \mathrm{~s}$ on the filaments ${ }^{10}$. We confirmed this fast exchange property of Lifeact by single-molecule speckle (SiMS) microscopy ${ }^{11-13}$. The dwell time of Atto 488-labeled Lifeact showed a single exponential decay with a half-life of 23 ms (Supplementary Fig. 1a). We acquired $2 \times 10^{5}$ SiMS images of Lifeact on actin filaments in vitro with 50-ms exposure times and acquisition intervals. The central position of each speckle was then identified using the DAOSTORM computer program $^{14}$ (for example, Supplementary Fig. 1b). Integration of the position information from numerous Lifeact speckles reconstructed an image of actin filaments (Fig. 1b and Supplementary Fig. 1c). The mean width of a single actin filament was $23 \mathrm{~nm}$ in full-width at half-maximum (FWHM) in the image reconstructed using only (top 12\%) high-brightness speckles (Fig. 1c and Supplementary Fig. 1d).

A major problem with current super-resolution microscopy is the difficulty of fully labeling the structure using antibodies and photoactivatable fluorescent proteins. We examined the fidelity of super-resolved images with different labeling densities by using the coefficient of variation of the labeling density along the actin filament, but not the Fourier ring correlation ${ }^{15}$ (Supplementary Note 1). Because actin subunits and antibodies are 6 and $12 \mathrm{~nm}$ wide, respectively, a single actin filament composed of 360 subunits/ $\mu \mathrm{m}$ can be labeled by an antibody at a maximum density of only 180 molecules/ $\mu \mathrm{m}$. Our IRIS image of an actin filament labeled at this density showed a highly discontinuous pattern along the filament (Fig. 1d, e). A $6.5 \times$ increase in the label density improved the continuity of the filament image, but the label intensity along the filament still fluctuated with a coefficient of variation of $38 \%$. A density of $1.2 \times 10^{4}$ labels/ $\mu \mathrm{m}$ using $2 \times 10^{5}$ frames further improved the homogeneity of the labeling distribution (coefficient of variation $=13 \%$ ) (Fig. 1d, e and Supplementary Fig. 1e). We also compared the images of microtubules obtained by IRIS (see below), STORM $^{16,17}$, nanobody-based labeling ${ }^{18}$ and Exchange-PAINT ${ }^{7}$. Among them, the IRIS image showed the most continuous label intensity along the microtubules (Supplementary Fig. 2). Thus, our IRIS method overcomes the labeling density problem that has hitherto hampered the resolution of two or three targets coexisting closely. 
In fixed and permeabilized cells, IRIS resolved two parallel actin bundles $50 \mathrm{~nm}$ apart (Fig. 1f, g). In contrast, the two actin bundles were not resolvable in the summed SiMS image, which represents images obtained by conventional immunofluorescence (Fig. 1g).

Drawing on the useful binding characteristics of the Lifeact probe, we have established a method to rapidly identify IRIS probes for other cell structures (Supplementary Note 2, Supplementary Table 1, Supplementary Figs. 3-5 and Online Methods). We successfully identified 18 IRIS probes (39\%) out of 46 fragments of 15 cytoskeleton- or focal adhesion-associated proteins. The super-resolved images of actin, microtubules, intermediate filaments and focal adhesions were reconstructed using Lifeact, a CLIP-170 fragment (residues 3-309), a plecin-1 fragment (residues 4022-4364) and a phosphatidylinositol-(4)-phosphate 5-kinase type I $\gamma$-90 (PIPKI $\gamma$ ) fragment (residues 641-668), respectively (Fig. 2 and Supplementary Video 1). The FWHM of microtubules was $41 \mathrm{~nm}$ in the IRIS image reconstructed using only high-brightness (top 12\%) speckles (Supplementary Fig. 6). Taking advantage of the exchangeability of IRIS probes, we sequentially acquired images of these IRIS probes on four different cytoskeletal structures. In addition, we investigated the three-dimensional (3D) networks of actin filaments, microtubules and intermediate filaments. We acquired images of each IRIS probe alternately by total-internal-reflection fluorescence (TIRF) imaging and epi-illumination microscopy; these images were then used to reconstruct super-resolution images at the bottom and in the entire peripheral area of the cell. The $z$ position (nm) of the structures was estimated by the signal ratio between IRIS images captured with TIRF microscopy and those captured with epi-illumination microscopy, which was calibrated with images of tilted fluorescent microtubules ${ }^{19}$ (Supplementary Figs. 7 and 8 and Online Methods). The data showed that actin arcs running parallel to the cell contour gradually rose toward the center above the radial actin bundles localized at the bottom (Fig. 2a). Microtubules and intermediate filaments behind the lamellipodium base were excluded from the cell bottom in several places (Fig. $2 \mathbf{b}, \mathbf{c}$ ).

The IRIS images allowed us to observe spatial relationships between multiple cytoskeletal structures at a resolution beyond the diffraction limit. In lamellar regions, intermediate filaments were often tangled with actin stress fibers but not with microtubules (Fig. 3a-c). The tangled actin and intermediate filaments appeared to be tethered to each other. Their cross-sectional profiles show that actin stress fibers overlapped with intermediate filaments (Fig. 3d, e). In peripheral regions, intermediate filaments did not tangle with actin stress fibers (Fig. 3f, g), whereas some intermediate 
filaments ran along microtubules (Fig. 3f, h). The cross-sectional profiles show that intermediate filaments are overlapped with microtubules but not with actin filaments (Fig. 3i, j). Thus, IRIS was capable of revealing area-specific proximity between four cytoskeletal components over the entire cell area.

Previous studies have reported functional interactions between microtubules, actin filaments and focal adhesions in their assembly and disassembly ${ }^{20,21}$. In IRIS images, we noticed that in the vicinity of focal adhesions and stress fibers, the height of microtubules locally changed from $100 \mathrm{~nm}$ to $200 \mathrm{~nm}$ (Supplementary Fig. 9a-d). Components of focal adhesions are located $30-80 \mathrm{~nm}$ above the glass surface ${ }^{22}$. The lifted microtubules may not have been in contact with focal adhesions but rather appeared to climb on actin stress fibers. We therefore investigated the movement of microtubule tips near focal adhesions and stress fibers by observing the live-cell dynamics of EB1-EGFP and then performing IRIS imaging after fixation. Our imaging system enables live-cell 3D imaging with acousto-optic tunable filters for fast switching between TIRF and epi-illumination microscopy. Superimposing the 3D trajectory of the microtubule tip on the super-resolved images revealed that when the growing microtubule tip contacted stress fibers, the tip moved upward, away from the underlying focal adhesions (Supplementary Fig. 9e and Supplementary Video 2). We also observed that microtubules often climbed on actin stress fibers and then changed direction laterally along the contour of the stress fibers (Supplementary Fig. 10 and Supplementary Video 3). In both cases, the growth of the EB1-labeled microtubule tip slowed down on contact with the stress fiber before changing direction (Supplementary Figs. 9e and 10b). These data suggest that the speed and direction of microtubule growth is highly influenced by the collision and subsequent interaction with actin stress fibers. The combination of IRIS with live-cell imaging can thus help dissect the formation process of multiple cytoskeletal structures that dynamically interact with each other.

Before we developed IRIS, an earlier work introduced an analogous method, PAINT $^{23}$. PAINT was shown to super-resolve the lipid bilayer morphology using a fluorescent dye, Nile-red, that rapidly shuttles between an aqueous solution and the lipid bilayer. Although the merits of PAINT in examining a large number of probe molecules was mentioned by Sharonov and Hochstrasser ${ }^{23}$, these authors did not test whether increasing the number of observations indeed improves the resolution, presumably because the labeling density problem became widely recognized a few years later ${ }^{5}$. High labeling density has been achieved for only a few targets such as DNA ${ }^{24}$. Thus, before our present study, the labeling density problem remained unsolved in super-resolution 
microscopy ${ }^{3,4,6}$. The labeling density of an actin filament by IRIS readily reached 60 times the maximum labeling density attainable with antibody binding. Our IRIS approach overcomes the labeling density problem that has deteriorated the fidelity of super-resolution microscopy.

In addition to surmounting the labeling density problem, the present study has demonstrated that protein-based exchangeable probes readily visualize multiple targets. Our data show the area-specific proximity of three cytoskeletons and focal adhesions with sub-diffraction-limit accuracy. In principle, the number of observable targets in IRIS is unlimited even if they coalesce in a narrow area-a crucial advantage over the existing methods. Furthermore, several IRIS probes for microtubules and focal adhesions imaged different parts of the structure (Supplementary Note 2 and Supplementary Figs. 4 and 5). The development of IRIS probes that bind to a molecule in a specific state may facilitate super-resolved mapping of the specific state of the molecule. IRIS - whose namesake is the Greek goddess of the rainbow — can shed light on the tangled jungle of the intracellular structures beyond the diffraction limit of light.

\section{ACKNOWLEDGEMENTS}

We thank H. Mizuno and S. Yamashiro (Kyoto University) for rabbit muscle actin and Y. Mimori-Kiyosue (RIKEN) for providing cDNAs encoding EB1, CLIP-170, CLASP $2 \gamma$ and APC. This work was supported by the Cabinet Office of the Government of Japan through the Funding Program for Next Generation World-Leading Researchers (LS013), by the Uehara Memorial Foundation (N.W.) and by Japan Society for the Promotion of Science (JSPS) KAKENHI grant numbers 26440091 and 15H01635 (T.K.).

\section{AUTHOR CONTRIBUTIONS}

T.K. and N.W. designed experiments and wrote the manuscript. T.K., M.H., A.T. and M.M. conducted experiments and analyzed the data.

\section{COMPETING FINANCIAL INTERESTS}

The authors declare no competing financial interests. 


\section{References}

1. Hell, S. W. Science 316, 1153-1158 (2007).

2. Huang, B., Babcock, H. \& Zhuang, X. Cell 143, 1047-1058 (2010).

3. Huang, B., Bates, M. \& Zhuang, X. Annu. Rev. Biochem. 78, 993-1016 (2009).

4. Sauer, M. J. Cell Sci. 126, 3505-3513 (2013).

5. Shroff, H., Galbraith, C. G., Galbraith, J. A. \& Betzig, E. Nat. Methods 5, 417-423 (2008).

6. Kanchanawong, P. \& Waterman, C. M. Methods Mol. Biol. 1046, 59-84 (2013).

7. Jungmann, R. et al. Nat. Methods 11, 313-318 (2014).

8. Betzig, E. et al. Science 313, 1642-1645 (2006).

9. $\quad$ Rust, M. J., Bates, M. \& Zhuang, X. Nat. Methods 3, 793-795 (2006).

10. Riedl, J. et al. Nat. Methods 5, 605-607 (2008).

11. Watanabe, N. \& Mitchison, T. J. Science 295, 1083-1086 (2002).

12. Higashida, C. et al. Science 303, 2007-2010 (2004).

13. Vale, R. D. Cell 135, 779-785 (2008).

14. Holden, S. J., Uphoff, S. \& Kapanidis, A. N. Nat. Methods 8, 279-280 (2011).

15. Nieuwenhuizen, R. P. et al. Nat. Methods 10, 557-562 (2013).

16. Bates, M., Huang, B., Dempsey, G. T. \& Zhuang, X. Science 317, 1749-1753 (2007).

17. Huang, B., Jones, S. A., Brandenburg, B. \& Zhuang, X. Nat. Methods 5, 1047-1052 (2008).

18. Ries, J., Kaplan, C., Platonova, E., Eghlidi, H. \& Ewers, H. Nat. Methods 9, 582-584 (2012).

19. Gell, C., Berndt, M., Enderlein, J. \& Diez, S. J. Microsc. 234, 38-46 (2009).

20. Small, J. V. \& Kaverina, I. Curr. Opin. Cell Biol. 15, 40-47 (2003).

21. Huda, S. et al. J. Cell Sci. 125, 5790-5799 (2012).

22. Kanchanawong, P. et al. Nature 468, 580-584 (2010).

23. Sharonov, A. \& Hochstrasser, R. M. Proc. Natl. Acad. Sci. USA 103, 18911-18916 (2006).

24. Schoen, I., Ries, J., Klotzsch, E., Ewers, H. \& Vogel, V. Nano Lett. 11, 4008-4011 (2011). 


\section{Figure Legends}

Figure 1. Super-resolution imaging using IRIS. (a) Outline of IRIS. Transient associations of single-molecule fluorescent probes with their targets are visualized, and their central positions are identified with nanometer accuracy. Integrating the position information from many frames produces a super-resolved image of the target. (b) Super-resolved image of a single actin filament in vitro by TIRF microscopy using Atto 488-Lifeact. Images are reconstructed from high-brightness speckles to improve the localization accuracy (Supplementary Fig. 1c). (c) Cross-sectional profile of single actin filaments ( $n=10$ filaments, gray bars). The black curve shows the Gaussian fit to the mean profile, with a FWHM of $23 \mathrm{~nm}$. Error bars, s.e.m. (d) Dependency of image quality on the labeling density in vitro. The labeling density of Atto 488-Lifeact per length is indicated. (e) Line profiles of the labeling density of Atto 488-Lifeact along the yellow outline in $\mathbf{d}$. The labeling density was normalized by the mean labeling density along the actin filament. (f) IRIS image of actin filaments in an XTC cell using Atto 488-Lifeact and TIRF microscopy. The image was reconstructed from $5 \times 10^{5}$ frames. (g) Comparison between the IRIS super-resolution image (upper left) and the summed SiMS image (lower left) of the boxed area in $\mathbf{f}$. The right graph shows cross-sectional profiles of two adjacent filaments between the yellow arrowheads in $\mathbf{g}$.

Figure 2. Super-resolution images of three cytoskeletons and focal adhesions. (a-d) Actin filaments (a), microtubules (b), intermediate filaments (c) and focal adhesions (d) were imaged using Lifeact, a CLIP-170 fragment, a plectin-1 (PLEC) fragment and a PIPKI $\gamma$ fragment, respectively. SiMS images of individual probes (except for the PIPKI $\gamma$ fragment) were acquired alternately by TIRF microscopy and epi-illumination (Epi) microscopy, and the super-resolved images in the cell bottom (TIRF) and the entire cell peripheral areas (Epi) were then reconstructed. In a-c, the TIRF image (green) and the Epi image (red) are merged. Actin arcs gradually rise toward the center (a, arrows). Microtubules and intermediate filaments are excluded from the bottom (arrows in $\mathbf{b}$ and $\mathbf{c}$ ). The enlarged images of the boxed area are shown on the right. The $z$ positions of the structures vary locally (see Supplementary Fig. 8 for detail). LP, lamellipodia; SF, stress fibers. (e) Merged image of the seven super-resolved images in a-d. Images were reconstructed from $2 \times 10^{5}$ frames (Lifeact), $4 \times 10^{4}$ frames (CLIP-170 fragment), $1.2 \times 10^{5}$ frames (PLEC fragment) and $4 \times 10^{4}$ frames (PIPKI $\gamma$ fragment). The mean labeling densities per $\mu \mathrm{m}^{2}$ in the whole cell area are $1.1 \times 10^{5}$ and $9.8 \times 10^{4}$ (Epi and TIRF images using Lifeact), $2.5 \times 10^{4}$ and $2.9 \times 10^{4}$ (Epi and TIRF images using the CLIP-170 fragment), $1.3 \times 10^{4}$ and $1.4 \times 10^{4}$ (Epi and TIRF images 
using the PLEC fragment) and $3.5 \times 10^{4}$ (TIRF image using the PIPKI $\gamma$ fragment).

Figure 3. Area-specific proximity between cytoskeletons and focal adhesions. (a-d) Super-resolved epi-illumination microscopy image of intermediate filaments (IF) (a), merged images of the intermediate filaments with actin filaments (Act) (b) or with microtubules (MT) (c) in the lamellar region of ROI1 in Figure 2e, and the triple-merged image of the boxed area in a (d). (e) Cross-sectional profiles of three cytoskeletons in $\mathbf{d}$ (between arrowheads). Intermediate filaments are tangled with actin stress fibers (arrows) but not with microtubules in the lamellar region. (f-i) Super-resolved TIRF image of intermediate filaments (f), merged images of the intermediate filaments with actin filaments (g) or with microtubules (h) in the peripheral region of ROI2 in Figure 2e, and the quadruple-merged image of the boxed area in $\mathbf{f}(\mathbf{i})$. (j) Cross-sectional profiles of three cytoskeletons in $\mathbf{i}$ (between arrowheads). The intermediate filaments overlap with a microtubule (arrow) but not with actin filaments and focal adhesions (FA) in the peripheral region. Scale bars in a and $\mathbf{f}$ apply to $\mathbf{b}, \mathbf{c}, \mathbf{g}$ and $\mathbf{h}$. 
a

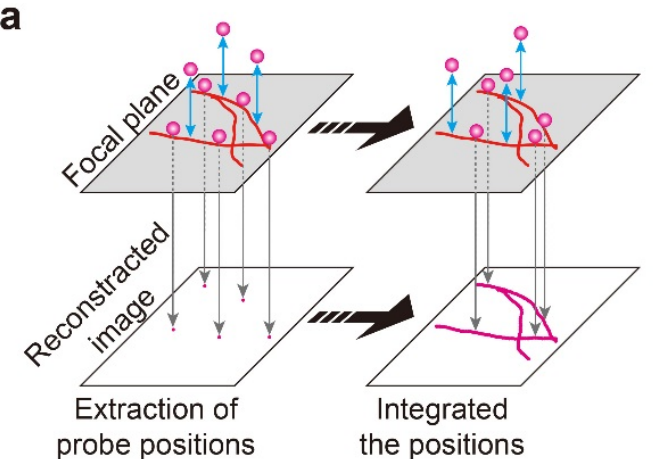

d

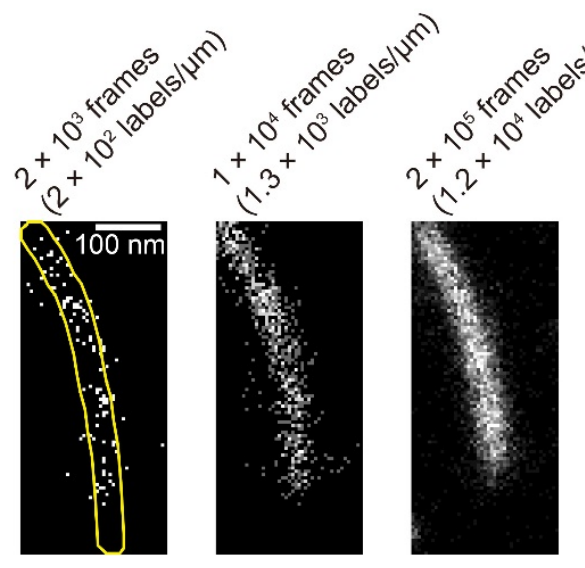

f

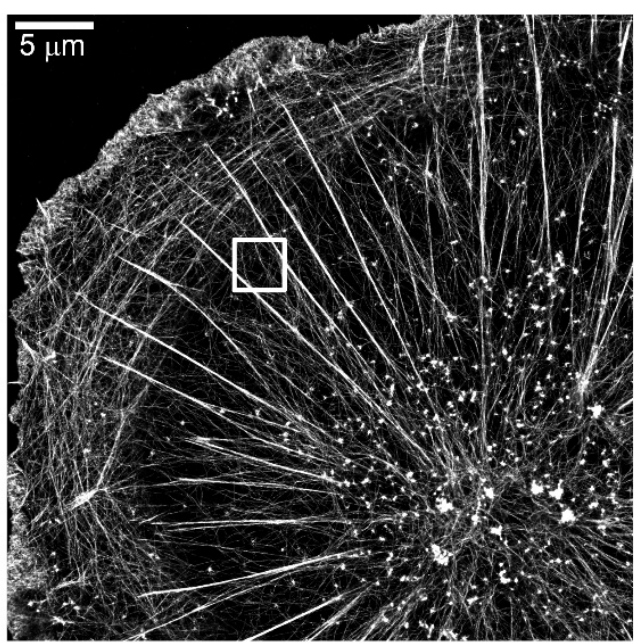

b

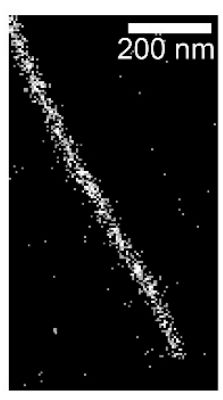

तो $e$

$-2 \times 10^{3}$ frames $-1 \times 10^{4}$ frames $-2 \times 10^{5}$ frames
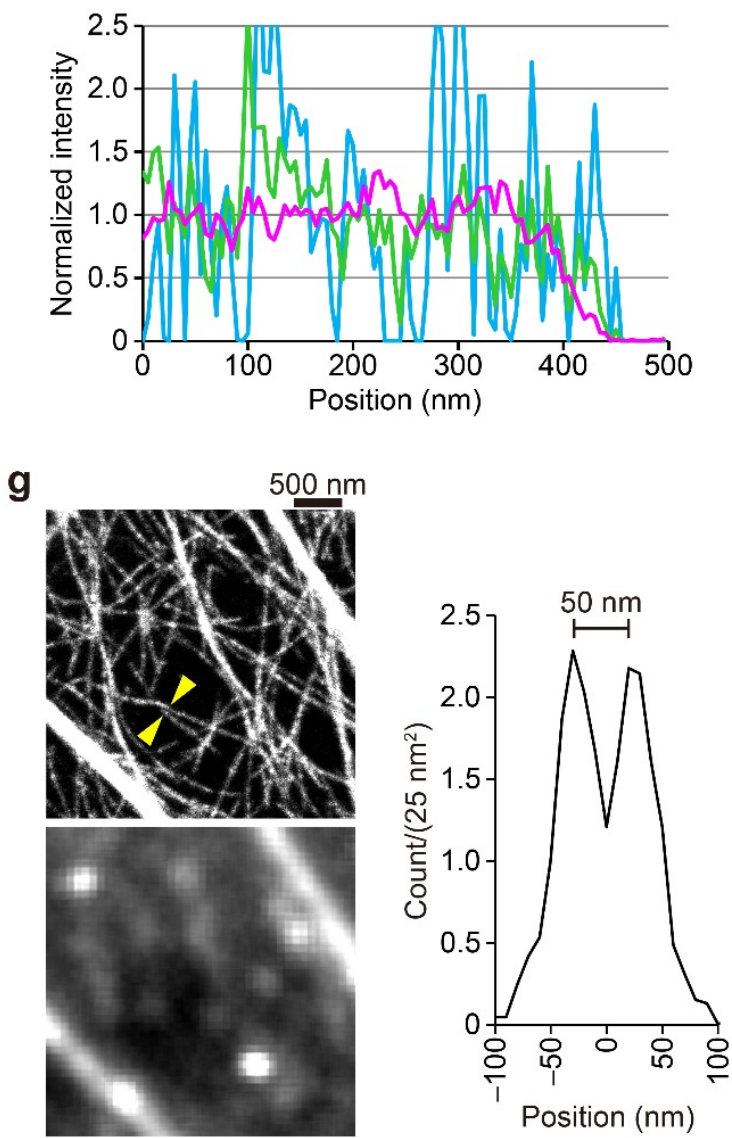

Fig.1 Kiuchi et al. 


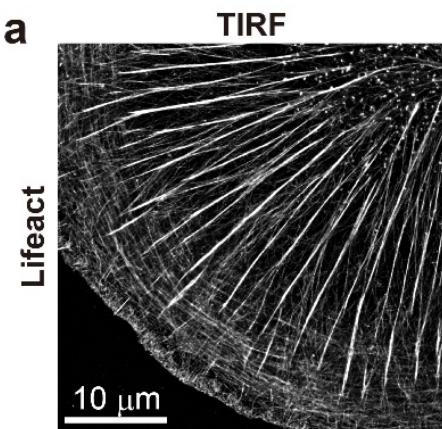

b
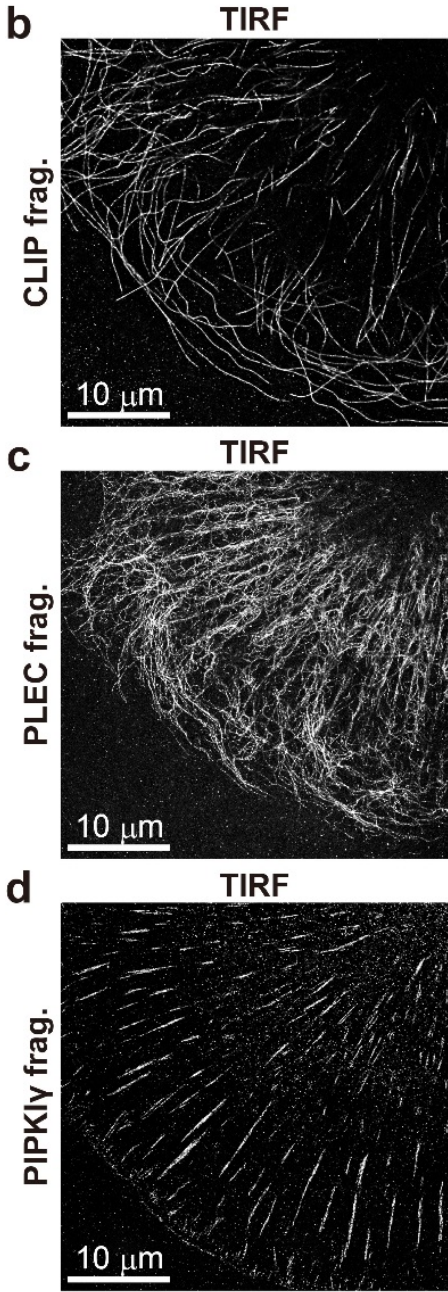

Epi

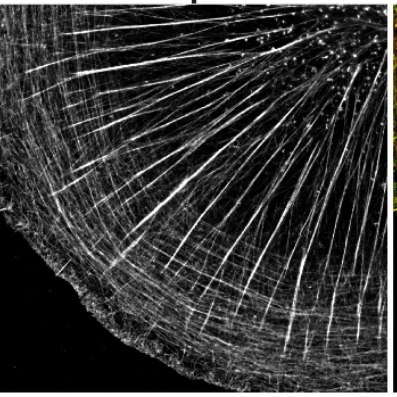

Epi

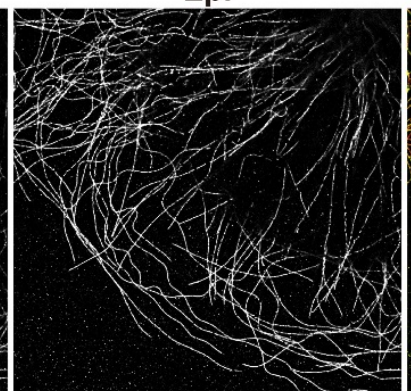

Epi

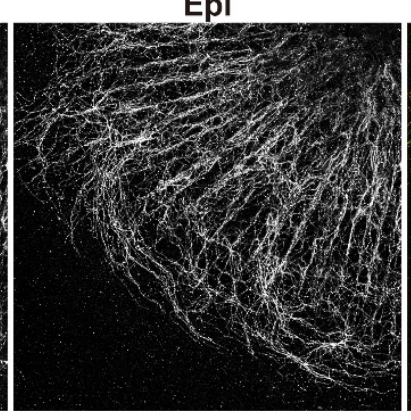

e

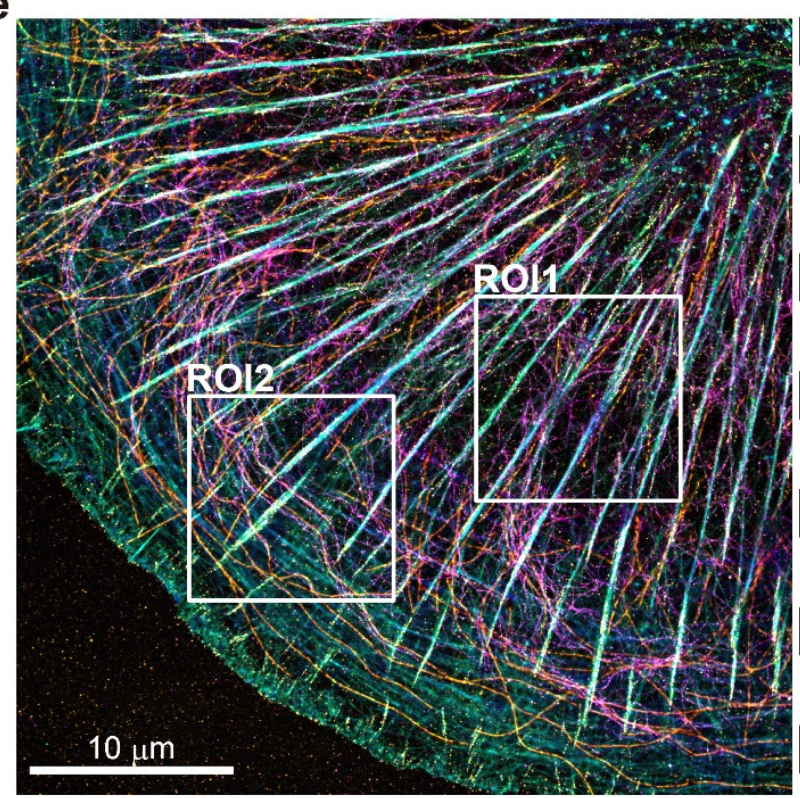

Fig.2 Kiuchi et al.
Merged
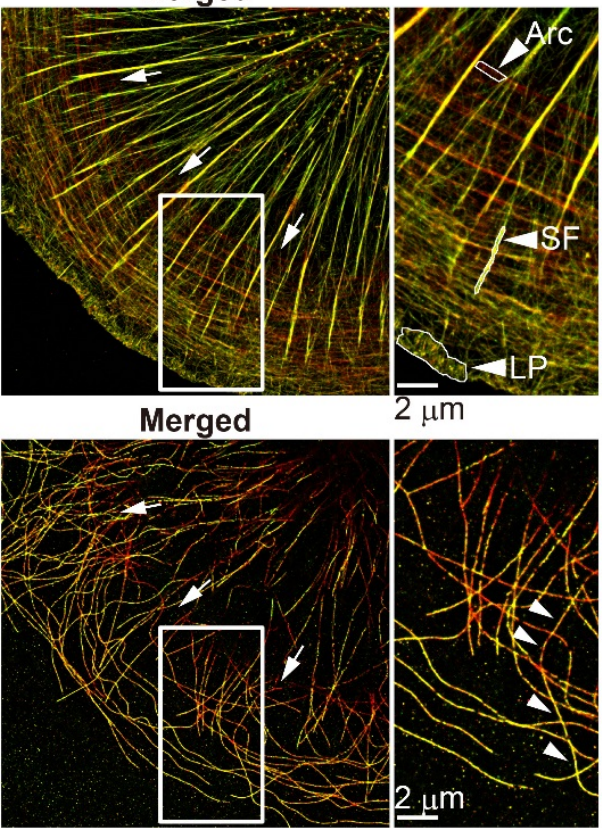

Merged

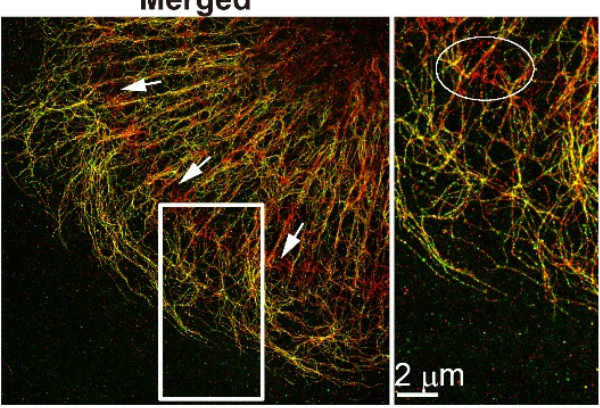

$\square$ Lifeact

Lifeact
(Epi)

$\square$ CLIP frag

CLIP frag

(Epi)

$\square \begin{aligned} & \text { PLEC frag } \\ & \text { (TIRF) }\end{aligned}$

$\square$ PLEC frag

$\square \begin{aligned} & \text { PIPKIy frag } \\ & \text { (TIRF) }\end{aligned}$ 
a

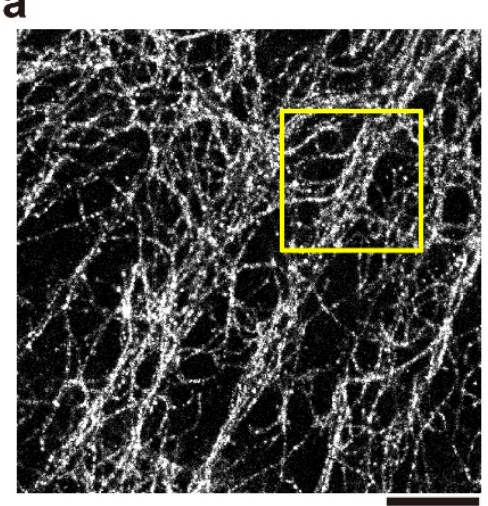

d

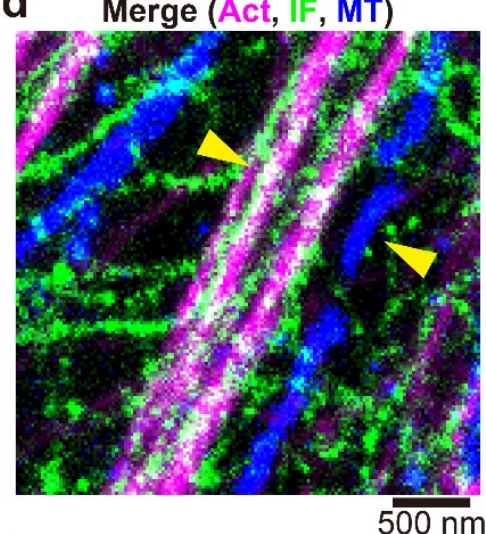

f

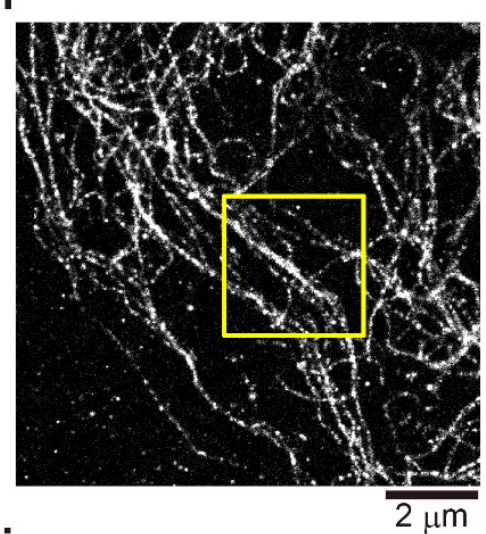

i Merge (Act, IF, MT, FA)

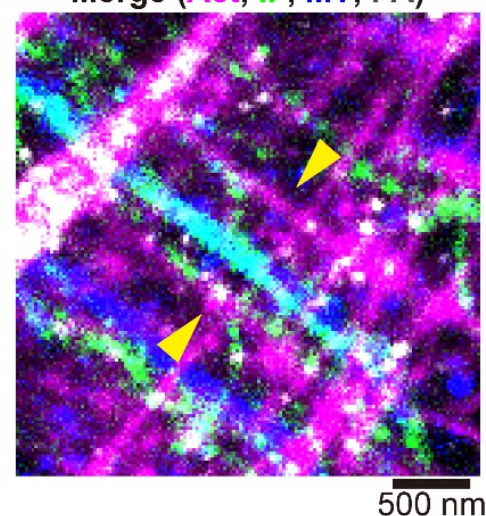

b

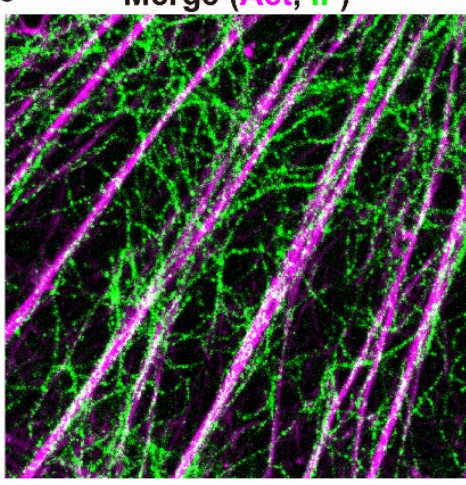

e

-Actin filament -Microtubule - Intermediate filament
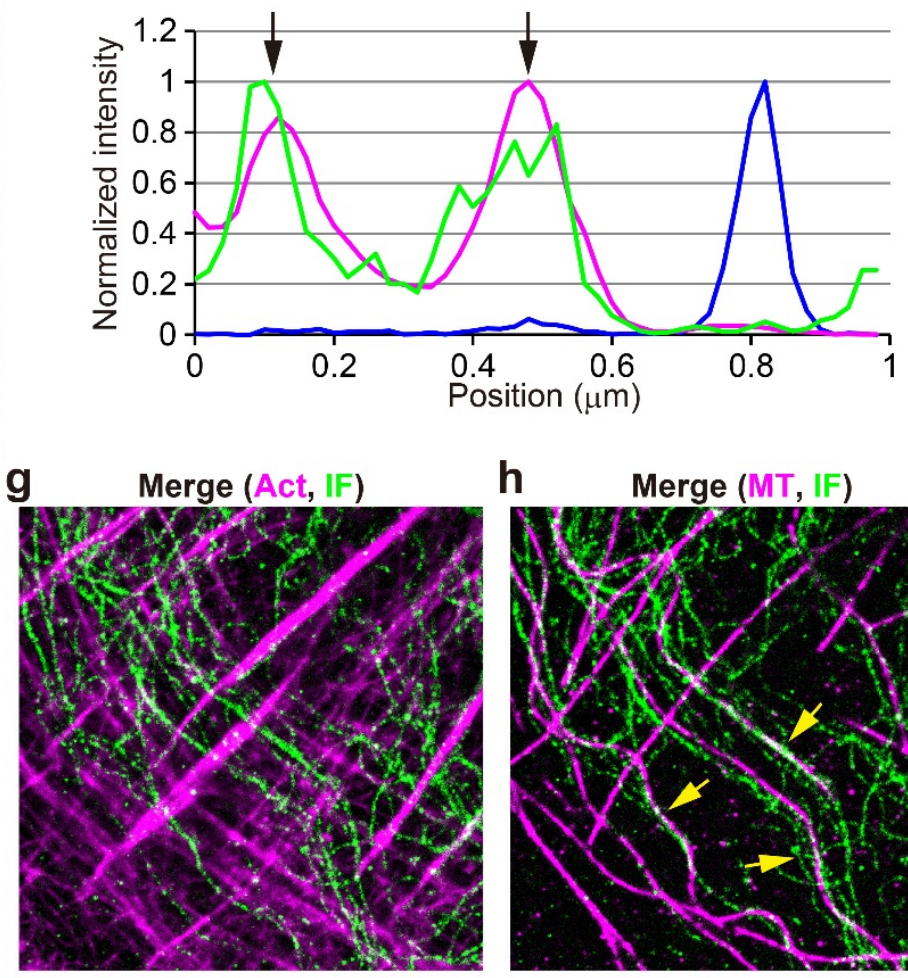

h Merge (MT, IF)

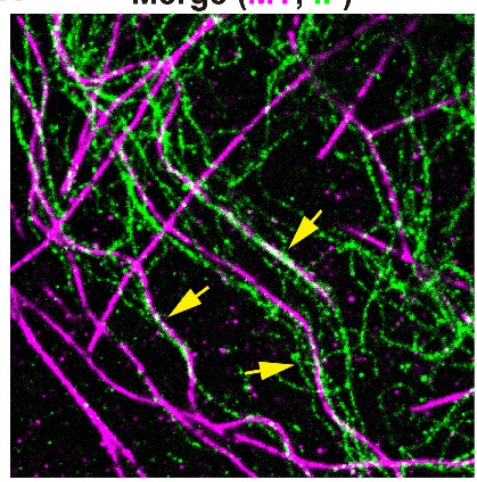

j

- Actin filament - Microtubule - Intermediate filament

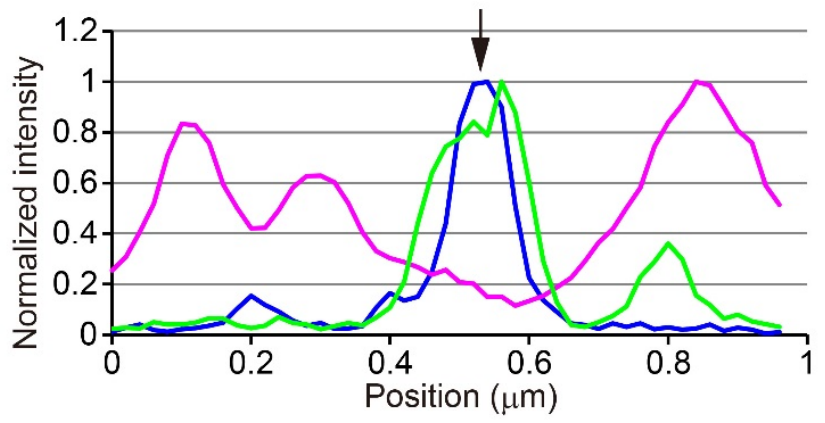

Fig.3 Kiuchi et al. 


\section{ONLINE METHODS}

\section{Plasmids and reagents.}

The expression plasmids encoding N-terminally FLAG-tagged EGFP (pFLAG-EGFP-C1) and C-terminally 3×FLAG-tagged EGFP (p3×FLAG-EGFP-N3) were constructed from the pEGFP-C1 and pEGFP-N3 vectors, respectively (Clontech Laboratories). EST clones encoding mouse MAP4, human Tau isoforms 3 and 4, mouse KIF1A, human plectin-1 and Xenopus laevis talin-1 were purchased from Open Biosystems. cDNA encoding human FAK was purchased from the DNASU Plasmid Repository. The GenBank/EMBL/DDBJ accession nos. for sequence data are as follows: BC055332 (MAP4), BC114948 (Tau isoform 3), BC101936 (Tau isoform 4), BC062891 (KIF1A), BM559026 (plectin-1), CF282569 (talin-1) and BC035404 (FAK). cDNAs encoding human EB1, rat CLIP-170, human CLASP2 $\gamma$ and human APC were provided by Y. Mimori-Kiyosue. The plasmids encoding human paxillin, chicken Src and human vinculin were previously described ${ }^{25-27}$. Each cDNA was inserted using PCR into the pFLAG-EGFP-C1 or p3×FLAG-EGFP-N3 vector (see Supplementary Table 1). The expression plasmid encoding the N-terminally FLAG-EGFP-tagged PIPKI $\gamma$ fragment (residues 641-668) was constructed by inserting a synthetic cDNA encoding PTDERSWVYSPLHYSAQAPPASDGESDT into the pFLAG-EGFP-C1 vector.

The synthesized Lifeact peptide (MGVADLIKKFESISKEE) with an N-terminal Atto 488 fluorophore was purchased from Sigma-Aldrich.

\section{Preparation and screening of protein-based exchangeable probes.}

To find exchangeable probes for super-resolution images of microtubules, intermediate filaments and focal adhesions, we tested proteins and their fragments that are known to localize at the target structures. Supplementary Table 1 shows the list of tested candidate molecules. Their expression plasmids for EGFP-fused molecules were constructed with reference to the literature in Supplementary Table 1. Human embryonic kidney (HEK)-293F cells (testing mycoplasma negative) were transfected with a plasmid encoding a candidate protein tagged with FLAG-EGFP or 3×FLAG-EGFP. After 3-4 d, the cells were lysed in lysis buffer (10 mM HEPES pH 7.2, $90 \mathrm{mM} \mathrm{KCl,} 3 \mathrm{mM} \mathrm{MgCl}$, 0.2\% Triton X-100, $100 \mu \mathrm{M}$ DTT) containing protease inhibitor cocktail (Nacalai Tesque). After centrifugation, the supernatant was collected. For the screening of IRIS probes, we examined the ability of the candidate probe in the supernatant to bind to the structure in paraformaldehyde (PFA)-fixed and permeabilized $X$. laevis XTC cells (testing mycoplasma negative). The emergence and disappearance 
of single-molecule speckles (SiMSs) on the structure were tested. The IRIS probes were screened on the basis of the following criteria: (i) the distribution of its target along the structure is detectable in the summed SiMS image; (ii) the probe can be washed out after SiMS imaging; (iii) the probe rapidly dissociates from the target $\left(\mathrm{T}_{1 / 2}=20-100 \mathrm{~ms}\right.$ and up to $500 \mathrm{~ms}$ ) (Supplementary Fig. 3); and (iv) integration of the central positions of the speckles can reconstruct an image of the target.

For IRIS experiments, the probes were overexpressed in HEK-293F cells and collected with anti-DYKDDDDK (FLAG) antibody beads (Wako). The beads were washed four times with an excess amount of HEPES-buffered solution (10 mM HEPES $\mathrm{pH}$ 7.2, $90 \mathrm{mM} \mathrm{KCl}, 3 \mathrm{mM} \mathrm{MgCl}$, $100 \mu \mathrm{M}$ DTT). The bound proteins were eluted with the HEPES-buffered solution containing $0.5 \mathrm{mg} / \mathrm{ml}$ DYKDDDDK (FLAG) peptide (Wako) or $3 \times$ FLAG peptide (Sigma-Aldrich) for $30 \mathrm{~min}$.

Eighteen candidates out of 46 fragments (39\%) of 15 cytoskeleton- or focal adhesion-associated proteins passed this screening process (Supplementary Table 1). By our current approach, IRIS targets are limited to only those with known binding partners. This problem can be overcome by using protein interaction assays such as phage display and the yeast two-hybrid system to screen the probes.

\section{Multicolor super-resolution imaging procedure by IRIS.}

XTC cells were cultured in 70\% Leibovitz's L15 medium supplemented with $10 \%$ fetal bovine serum. A multicolor super-resolution image was produced from a large number of fluorescence SiMS images, which were sequentially acquired in a fixed and permeabilized XTC cell (20,000-500,000 frames per probe) with different exchangeable probes. The cells were allowed to spread on a $0.1 \mathrm{mg} / \mathrm{ml}$ poly(l-lysine)and $10 \mu \mathrm{g} / \mathrm{ml}$ fibronectin-coated coverslip in 70\% Leibovitz's L15 medium without serum to observe distinct stress fibers and focal adhesions ${ }^{27}$. After $2 \mathrm{~h}$, the cells were fixed and permeabilized with 3.7\% PFA and 0.5\% Triton X-100 in a cytoskeleton buffer (10 mM MES pH 6.1, $90 \mathrm{mM} \mathrm{KCl,} 3 \mathrm{mM} \mathrm{MgCl} 2,2 \mathrm{mM}$ EGTA) for $20 \mathrm{~min}$. After a blocking step with $4 \%$ bovine serum albumin for $30 \mathrm{~min}$, the cells were subjected to the purified IRIS probe in an imaging solution comprising the HEPES-buffered solution with an oxygen scavenging mix (200 $\mu \mathrm{g} / \mathrm{ml}$ glucose oxidase, $35 \mu \mathrm{g} / \mathrm{ml}$ catalase, 4.5 $\mathrm{mg} / \mathrm{ml}$ glucose, $0.5 \%$ 2-mercaptoethanol $)^{28}$. The concentration of the probe was 1-100 $\mathrm{nM}$. Without the oxygen scavenging mix, laser-induced photodamage was apparent after several tens of thousands of SiMS images were acquired.

The concentration of IRIS probes during image acquisition is determined by the density of SiMSs so that individual speckles can be identified on the target structures. 
The density of the speckles [S] is proportional to the concentration of the fluorescent IRIS probe bound to the target, [FT], as,

$$
[\mathrm{S}] \propto[\mathrm{FT}]=[\mathrm{F}][\mathrm{T}] / K d
$$

where [F], [T] and $K d$ are the concentrations of the fluorescent IRIS probe and the unlabeled target, and the dissociation constant for the complex, respectively. $[\mathrm{F}]$ is the product of the probe concentration and the labeling efficiency. Atto 488-Lifeact was used at a peptide concentration of $1-2.4 \mathrm{nM}$ in part owing to the high abundance of its target, actin. At the higher concentration of the Lifeact probe, speckles tended to overlap with each other and the accuracy of the probe localization was diminished.

Another factor that may limit the probe concentration is the ratio between the signal intensity of SiMSs and the background fluorescence. In the case of the PIPKI $\gamma$ fragment, the probe was used at $84 \mathrm{nM}$ to maximize the number of speckles per frame under the TIRF illumination. Under the epi-illumination, however, the probe concentration needed to be decreased to reduce the background fluorescence derived from out-of-focus probes. The appropriate concentration of each IRIS probe should be empirically determined by trials using fixed-cell specimen.

The SiMS images were acquired using an inverted microscope (Olympus IX83-ZDC) equipped with an Olympus PlanApo 100×/1.45-numerical aperture (NA) objective lens, a $2 \times$ intermediate lens and an electron-multiplying charge-coupled device (EMCCD) camera (Evolve 512, Roper) controlled by MetaMorph software (Molecular Device). Its focus was automatically maintained at the bottom of the cell by a $z$ drift compensation system during the long-term imaging. The IRIS probe was alternately excited with a 473-nm laser line $(50 \mathrm{~mW})$ for epi-illumination microscopy and a 488-nm laser line (50 $\mathrm{mW}$ ) for TIRF microscopy. In the epi-illumination mode, the incidence angle of the 473-nm laser line was tilted to reduce background fluorescence from out-of-focus unbound probes. The epi- and TIRF-illuminated images were used to estimate the $z$ position of each object (see below). Image acquisition was performed by repeating the following set of procedures: (i) bright-field image acquisition, (ii) epi-illuminated SiMS imaging (frame rate: $20 \mathrm{~Hz}, 250$ frames) and (iii) TIRF-illuminated SiMS imaging (frame rate: $20 \mathrm{~Hz}, 250$ frames). The bright-field image was used to correct the drift of the microscope stage in the lateral dimension (see below). In the case of the observation of focal adhesions, epi-illuminated SiMS imaging was omitted and TIRF-illuminated imaging was performed (frame rate: $20 \mathrm{~Hz}, 500$ frames). Each set of procedures took $27 \mathrm{~s}$ and was repeated 160-240 times (CLIP-170 
fragment for microtubules), 40 times (PIPKI $\gamma$ fragment, paxillin and Src fragment for focal adhesions), 800 times (Lifeact for actin filaments) or 400-480 times (plectin-1 fragment for intermediate filaments). For maintenance of the oxygen-scavenging capacity in the imaging solution, each imaging solution with the probe was replaced with fresh solution every 40 sets for the CLIP-170 fragment, the PIPKI $\gamma$ fragment, paxillin and the Src fragment and every 80 sets for Lifeact and the plectin- 1 fragment. For multicolor imaging of three cytoskeletons and focal adhesions, SiMS imaging was carried out in the following order: the CLIP-170 fragment, the PIPKI $\gamma$ fragment (or Src fragment and paxillin), Lifeact and the plectin-1 fragment. After SiMS imaging of each probe, the probe was washed out ten times with the HEPES-buffered solution. The remaining fluorescence of the probe was completely photobleached in the HEPES-buffered solution with the oxygen scavenging mix, and another probe was then loaded. In Figure 1 and Supplementary Figure 6, Atto 488-Lifeact and the CLIP-170 fragment were excited by the simultaneous illumination of 473-nm and 488-nm laser lines to obtain strong signals from each speckle.

For the imaging of actin filaments in vitro, monomeric actin was prepared from rabbit skeletal muscle as previously described ${ }^{27,29,30}$. Phalloidin-stabilized F-actin was observed on a $1 \mathrm{mg} / \mathrm{ml}$ poly(l-lysine)-coated coverslip in the imaging solution. The stage drift was corrected with the bright-field images of nonfluorescent beads (see below).

\section{Image reconstruction procedure in IRIS.}

A super-resolution image was reconstructed by plotting the central points of each fluorescent speckle on a blank image with subpixel accuracy. The number of plotted points was typically $10^{6}-10^{8}$. The subpixel-accuracy central point was estimated by fitting a point spread function (PSF) of this microscope using the DAOSTORM computer program ${ }^{14}$. To correct the stage drift, we determined the drift distance by the following autocorrelation function, $A_{N}\left(x_{\text {drift, }}, y_{\text {drift }}\right)$, of the bright-field images acquired at each set of imaging procedures:

$A_{N}\left(x_{\text {drift }}, y_{\text {drift }}\right)=\sum_{\mathrm{y}=\mathrm{y}_{0}}^{\mathrm{y}_{\mathrm{m}}} \sum_{\mathrm{x}=\mathrm{x}_{0}}^{\mathrm{x}_{\mathrm{m}}}\left[\mathrm{I}_{0}(\mathrm{x}, \mathrm{y}) \times \mathrm{I}_{\mathrm{N}}\left(\mathrm{x}+\mathrm{x}_{\text {drift }}, \mathrm{y}+\mathrm{y}_{\text {drift }}\right)\right]$

where $x_{\text {drift }}$ and $y_{\text {drift }}$ are drift distances in the direction of the $x$ axis and $y$ axis, respectively. $I_{0}(x, y)$ and $I_{N}\left(x+x_{\text {drift, }} y+y_{\text {drift }}\right)$ are intensities at the pixel positions $(x, y)$ and $\left(x+x_{\text {drift }} y+y_{\text {drift }}\right)$ in the bright field images obtained in the 1 st and Nth sets, respectively. The product of $I_{0}(x, y)$ and $I_{N}\left(x+x_{\text {drift, }} y+y_{\text {drift }}\right)$ is accumulated within a 
region of interest in the bright field image. $A_{N}\left(x_{\text {drift, }}, y_{\text {drift }}\right)$, which is a function of the variables $x_{\text {drift }}$ and $y_{\text {drift, }}$ will be maximal if the two bright field images coincide. The $x_{\text {drift }}$ and $y_{\text {drift }}$ values that maximize $A_{N}\left(x_{\text {drift }}, y_{\text {drift }}\right)$ were computed using a customized plug-in in ImageJ software (http://rsb.info.nih.gov/ij/). First, the drift of the bright field images in the Nth set was corrected using the $x_{\text {drift }}$ and $y_{\text {drift }}$ values with pixel accuracy. To further determine the drift distance with subpixel accuracy, we enlarged the bright-field image and the corrected image in the first and $N$ th set using a bicubic method. Using the $A_{N}\left(x_{\text {drift }}, y_{\text {drift }}\right)$ of the enlarged images, we determined the drift distances with subpixel accuracy. The central positions of speckles in the SiMS images in the Nth set were corrected with the drift distances. Plotting the corrected central positions produced a super-resolution image. In addition, the positions of speckles continuously observed in more than 10 or 20 frames were excluded from the reconstructed images of Lifeact or the plectin-1 fragment. The two probes occasionally stuck to the targets when the strong excitation laser power was used.

\section{Image processing for mapping the $z$ positions of objects.}

Because the TIRF excitation intensity exponentially decays away from the coverglass, the height of the object was estimated from the ratio of its TIRF-illuminated image and its epi-illuminated image. The $z$ position from the coverglass surface was calibrated by the previous method using tilted fluorescent microtubules with respect to the coverglass in a low-melting-point agarose gel ${ }^{19}$. HyLight 488-labeled tubulin was purchased from Cytoskeleton. The labeled microtubules were prepared according to a previous report ${ }^{19}$. The tilted microtubules, one end of which touched the coverglass, were imaged by TIRF and epi-illumination microscopy. Epi-illuminated images were acquired as a $z$-stack image (0.2 $\mu \mathrm{m}$ step size) (Supplementary Fig. 7a). In the intensity line profile along the epi-imaged microtubule, the $x y$ position of the highest intensity was used to determine the intersection of the tilted microtubule and the focal plane (Supplementary Fig. 7a, yellow arrowheads). By connecting the intersections among the $z$-stacked images, we determined the $z$ distance of each point along the tilted microtubule. The $z$ profile of the TIRF excitation intensity was characterized by associating the $z$ distance of each point with the ratio of the intensity of the TIRF-illuminated microtubule and that of the epi-illuminated microtubule at the bottom (Supplementary Fig. 7b). The $z$ profile was fitted with a single exponential decay function (Supplementary Fig. 7b, black line). The $z$ positions were rescaled by a factor of 0.82 to account for the difference in the refractive index between the immersion oil and the imaging solution ${ }^{19}$. The inverse function of the exponential function was used to determine the $z$ position of 
an object as follows:

$\mathrm{z}=-\alpha_{\mathrm{z}} \ln \left(\beta \frac{\mathrm{F}^{\mathrm{TIRF}}}{\mathrm{F}^{\mathrm{Epi}}}\right)$

where $\alpha_{z}$ is the $z$ position at which the intensity of TIRF illumination is $1 / \mathrm{e}, \beta$ is a parameter to correct for the difference in laser power between TIRF and epi-illumination microscopy, and $F^{\mathrm{TIRF}}$ and $F^{\mathrm{Epi}}$ are the fluorescence intensities of the object in TIRF and epi images, respectively. The $z$ position maps of three cytoskeletons were converted from the ratio image of their TIRF-illuminated IRIS image to their epi-illuminated IRIS image. For this purpose, the peak intensity of the fluorescence speckle was also fitted using DAOSTORM. The IRIS images were reconstructed by plotting the peak intensity at the central position of the speckle. The z-position map was masked by thresholding the fluorescence intensities in the summed image of the TIRFand epi-IRIS image to remove noise in regions without the cytoskeletons. In layered structures such as actin stress fibers, the calculated $z$ position represents the centroid height of the structure along the $z$ axis.

The $z$ position of the traced microtubule plus end by live-cell imaging of EB1-EGFP was converted from the ratio of the mean intensity in a 0.4 - $\mu$ m-diameter region of the plus end in the TIRF image to that in the epi image.

\section{Live-cell imaging of the movement of microtubule plus ends.}

XTC cells were transfected with an expression plasmid for EGFP-fused EB1. After 3-4 d, time-lapse imaging of EB1-EGFP was carried out in the cells at 1-s intervals. At each time point, two fluorescence images were alternately acquired using TIRF and epi-illumination microscopy with a $100 \mathrm{~ms}$ exposure time. We traced EB1-labeled microtubule plus ends using an ImageJ plug-in, Speckle TrackerJ ${ }^{27,31}$. The $z$ position of the traced microtubule plus end was calculated as described above. The local speed of the plus end was calculated with a linear approximation of its $x y$ positions at five consecutive time points. 


\section{References}

25. Yamana, N. et al. Mol. Cell Biol. 26, 6844-6858 (2006).

26. Tanji, M. et al. Mol. Cell Biol. 30, 4604-4615 (2010).

27. Yamashiro, S. et al. Mol. Biol. Cell 25, 1010-1024 (2014).

28. Desai, A., Verma, S., Mitchison, T. J. \& Walczak, C. E. Cell 96, 69-78 (1999).

29. Mizuno, H. et al. Science 331, 80-83 (2011).

30. Mizuno, H. \& Watanabe, N. Methods Enzymol. 540, 73-94 (2014).

31. Smith, M. B. et al. Biophys. J. 101, 1794-1804 (2011). 